\title{
The "crushing" aortic prosthesis: a fatal acute myocardial infarction
}

\begin{abstract}
We report a 67 years old woman with prior mitral and aortic metallic prosthesis 14 days before, who came to the Emergency Room (ER) due to chest pain and hemodynamic instability, with dyspnoea in NYHA III and with an echocardiography showing hypocinesia of the anterior wall of the heart with low Left Ventricular Ejection Fraction (LVEF) of $35 \%$ and both prosthesis with normal function. The electrocardiogram showed sinus tachycardia and diffuse ST depression, diagnosing of Non ST-Elevation Myocardial Infarction (NSTEMI). We decided to perform an emergent coronary angiography. She had previously carried out a coronary angiography, five months earlier, with no evidence of Coronary Artery Disease (CAD). At the moment of the present NSTEMI, we observed high prosthesis implantation, with slow hemi-disc movement and evidence of thrombus in the Left Main (LM) with TIMI flow I, as well as in the left coronary sinus. An Intra-aortic Balloon pump was implanted and a thrombus aspiration catheter was used to improve the coronary flow. Then the TIMI flow improved, and we decided to fix the lesion with a $4.0 \times 15 \mathrm{~mm}$ drug eluting stent of everolimus (SYNERGY ${ }^{\mathrm{TM}}$ Bioabsorbable Polymer Drug-Eluting Stent System from Boston Scientific) in the ostia of LM. The stent was implanted without complications, but few minutes later the patient went into refractory cardiogenic shock. The hemi disc of the prosthesis was literally crushing the stent and the TIMI flow was progressively worsen with associated thrombus. Finally the patient was exitus because of cardiac arrest with no response to the advance cardiopulmonary resuscitation. We present the report case and a brief review of the literature.
\end{abstract}

Keywords: Acute coronary syndrome; Cardiac valve prosthesis; Coronary stent

\section{Case Report}

We report a 67 years old woman with prior mitral and aortic metallic prosthesis 14 days before, who came to the Emergency Room (ER) due to chest pain and hemodynamic instability, with dyspnoea in NYHA III. The echocardiography showed hypocinesia of the anterior wall of the heart with low Left Ventricular Ejection Fraction (LVEF) of $35 \%$ and both prosthesis with normal function. The electrocardiogram showed sinus tachycardia and diffuse ST depression, diagnosing of Non ST-Elevation Myocardial Infarction (NSTEMI). The laboratory showed hemoglobin of $11.4 \mathrm{~g} / \mathrm{dl}$, leukocytes of 9400 and an INR of 2.8, D-dimer of 1610 and Fibrinogen of 697. Arterial gases were: $\mathrm{pH} 7.47$ (7.35-7.45), $\mathrm{pCO}_{2} 32.7$ $\mathrm{mmHg}$ (32.0-48.0), $\quad \mathrm{PO}_{2} \quad 68.2 \mathrm{mmHg}$ (83.0-108.0), $\mathrm{O}_{2}$ saturation (estimated) $94.8 \%$. Blood glucose was $107.0 \mathrm{mg} / \mathrm{dL}$ $\begin{array}{lccc}(74.0-106.0), & \text { Urea } & 57.0 & \mathrm{mg} / \mathrm{dL} \\ (15.0-38.5), & \text { Creatinine } & 0.91 & \mathrm{mg} / \mathrm{dL}\end{array}$
(0.6-1.0), Ion Sodium $141.0 \mathrm{mEq} / \mathrm{L}$ (136.0-145.0), Ion Potassium $3.5 \mathrm{mEq} / \mathrm{L}$ (3.5-5.1), CK 31.0 U/L (26.0-192.0). Cardiac markers: Troponin I $0.056 \mathrm{ng} / \mathrm{mL}$ (0.0-0.06). The patient was correctly taking the medication from discharge, including anticoagulation with warfarin. She had previously carried out a coronary angiography, five months earlier, with no evidence of Coronary Artery Disease (CAD) (Figure 1A).

We decided to perform an emergent coronary angiography. We used 5000 IU of unfractionated Heparin and bolus and perfusion of abciximab adjusted for weight and renal function. At the coronary angiography, we observed high prosthesis implantation, with slow hemi-disc movement and evidence of thrombus in the Left Main (LM) with TIMI flow I, as well as in the left coronary sinus (Figure 1B). An Intra-aortic Balloon pump was implanted and thrombus aspiration catheter was used
Jeremias Bayon*, Melisa Santas-Alvarez, Raymundo Ocaranza-Sanchez and Carlos Gonzalez-Juanatey

Department of Cardiology, Hospital Universitario Lucus Augusti, Lugo, Galicia, Spain *Author for correspondence: jerebayon@gmail.com Tel: +034982296000 Submitted: 17 March 2017 Accepted: 04 April 2017 Published online: 10 April 2017 
to improve the coronary flow. We decided to fix the lesion with a $4.0 \times 16 \mathrm{~mm}$ drug eluting stent of everolimus (SYNERGY ${ }^{\text {Tn }}$ Bioabsorbable Polymer DrugEluting Stent System from Boston Scientific) in the ostia of LM (Figure 1C). The stent was implanted without complications, but few minutes later, patient went into refractory cardiogenic shock. The hemi-disc of the prosthesis was literally crushing the stent and the TIMI flow was progressively worsen with associated thrombus (Figure 1D). Finally the patient was exitus because of cardiac arrest with no response to the advance cardiopulmonary resuscitation.

Acute prosthetic valve thrombosis (PVT) is a serious complication with important morbidity and mortality [1]. Aortic infective endocarditis is associated with AMI from embolic vegetation [2] but it is less common embolic events from PVT, frequently with fatal outcome due to acute valve prosthesis dysfunction and myocardial ischemia. It is well known how the glycaemic control may affect prognosis on acute coronary syndrome after percutaneous coronary interventions [3] as well as correct anticoagulation in patients with metallic prosthesis. Proper treatment is not well established, but it looks appropriated to perform percutaneous revascularization when feasible or Emergent Surgery especially when the prosthesis is dysfunctional. Recently a short series of three patients with NSTEMI who were successfully treated with thrombolytic therapy was published [4]. Because of the lack of cases, more studies are necessary to set an adequate management of this pathology.

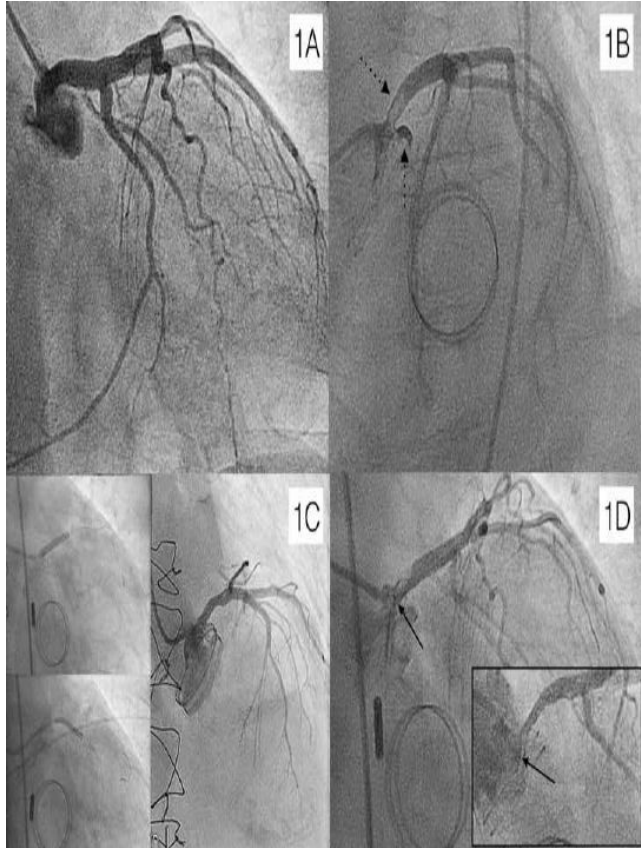

Figure 1: A) Coronariography prior to surgical valves replacement showing no evidence of coronary artery disease (CAD). B) Coronariography showing thrombus in the Left Main (LM) and in the Left coronary Aortic sinus (discontinued arrows). C) Coronary angioplasty with Drug Eluting Stent with TIMI flow III. D) Hemi-disc of the Aortic metallic prosthesis crushing LM stent with thrombus inside (continued arrows).

We report a 67 years old woman with prior mitral and aortic metallic prosthesis 14 days before, who came to the Emergency Room (ER) due to chest pain and hemodynamic instability, with dyspnoea in NYHA III and with an echocardiography showing hypocinesia of the anterior wall of the heart with low Left Ventricular Ejection Fraction (LVEF) of 35\% and both prosthesis with normal function.

The electrocardiogram showed sinus tachycardia and diffuse ST depression, diagnosing of Non ST-Elevation Myocardial Infarction (NSTEMI). We decided to perform an emergent coronary angiography. She had previously carried out a coronary angiography, five months earlier, with no evidence of Coronary Artery Disease (CAD). At the moment of the present NSTEMl, we observed high prosthesis implantation, with slow hemi-disc movement and evidence of thrombus in the Left Main (LM) with TIMI flow I, as well as in the left coronary sinus.

An Intra-aortic Balloon pump was implanted and a thrombus aspiration catheter was used to improve the coronary flow. Then the TIMI flow improved, and we decided to fix the lesion with a $4.0 \times 15 \mathrm{~mm}$ drug eluting stent of everolimus (SYNERGYTM Bioabsorbable Polymer Drug-Eluting Stent System from Boston Scientific) in the ostia of LM. The stent was implanted without complications, but few minutes later the patient went into refractory cardiogenic shock. The hemi disc of the prosthesis was literally crushing the stent and the TIMI flow was progressively worsen with associated thrombus.

Finally the patient was exitus because of cardiac arrest with no response to the advance cardiopulmonary resuscitation. We present the report case and a brief review of the literature.

\section{References}

1. ESC/EACTS. Guidelines on the management of valvular heart disease. Euro. Heart. J. 33: 2451-2496 (2012).

2. ESC. Guidelines for the management of infective endocarditis. Euro. Heart. J. 36: 3075-3123 (2015).
3. Marfella R. Peri-procedural tight glycemic control during early percutaneous coronary intervention up-regulates endothelial progenitor cell level and differentiation during acute ST-elevation myocardial infarction: effects on myocardial salvage. Int. J. Cardiol. 168(4): 3954-3962 (2013). 
4. Karakoyun S. A case series of prosthetic heart valve thrombosis-derived coronary embolism. Turk. Kardiyol. Dern. Ars. 42(5): 467-471 (2014). 